\title{
YAP regulates ALDHIAI expression and stem cell property of bladder cancer cells
}

This article was published in the following Dove Press journal:

OncoTargets and Therapy

\author{
Ai-Yue Zhao' \\ Yi-Jun Dai' \\ Jian-Feng Lian² \\ Yan Huang' \\ Jian-Guang Lin' \\ Yang-Bin Dai' \\ Tian-Wen $X u^{\prime}$ \\ 'Department of Medical Oncology, \\ the Second Affiliated Hospital of \\ Fujian Medical University, Quanzhou, \\ China; ${ }^{2}$ Department of Anesthesiology, \\ Quanzhou Children's Hospital, \\ Quanzhou 362000, China
}

Background: Yes-associated protein (YAP), a key player of the Hippo pathway, has been identified to have more and more important roles in tumorigenesis and may be an important biomarker for cancer therapy. YAP is important for bladder cancer cell migration, metastasis, and drug resistance; however, its function in bladder cancer stem cells remains unknown.

Purpose: The aim of this work was to examine the expression and role of YAP in bladder cancer stem cells.

Materials and methods: We identified that the expression level of YAP was significantly enriched in bladder cancer stem cells compared to noncancer stem cell population. Moreover, the effect of YAP on stem cell self-renewal was examined in bladder cancer cells by siRNA silencing approach. In addition, we showed that YAP is required for aldehyde dehydrogenase activity in bladder cancer cells.

Results: RNAseq analysis and quantitative real-time PCR results showed that silencing of YAP inhibited the expression of ALDH1A1 gene.

Conclusion: Collectively, our findings for the first time elucidated that YAP serves as a cancer stem cell regulator in bladder cancer, which provided a promising therapy strategy for patients with bladder cancer.

Keywords: bladder cancer, YAP, ALDH1, cancer stem cells

\section{Introduction}

Bladder cancer is the most common malignancy of the urinary system. Current standard treatment for muscle-invasive bladder cancer is surgery along with platinum-based chemotherapy. ${ }^{1}$ However, still over $30 \%$ of bladder cancers managed by surgery recur and progress to locally invasive or metastatic stages. ${ }^{2}$ Therefore, there is an unmet need for novel treatment to target bladder cancer. Cancer stem cells are rare population with stem cell characteristics, which play important roles in cancer chemoresistance, metastasis, and recurrence. ${ }^{3}$ Understanding the mechanism under cancer stem cell regulation could lay the groundwork for design of effective treatment.

Mounting evidence has shown the existence of cancer stem cells in solid tumors. The common used markers, such as CD44, Bmi1, CD133, ALDH1, and ABCG2, are often used for isolation of cancer stem cells from cell lines. ${ }^{4-6}$ In bladder cancer cell lines, aldehyde dehydrogenase high ( $\left.\mathrm{ALDH}^{\text {high }}\right)$ populations display resistance to cisplatin, increased colony-forming ability, migration, invasion, and ability to differentiate. ${ }^{7}$ However, the regulation of ALDH is very limited.

Yes-associated protein (YAP) and transcriptional co-activator with PDZ-binding motif (TAZ) are the key players of the Hippo pathway, which are involved in organogenesis and tumorigenesis. ${ }^{8}$ YAP has been increasingly demonstrated to play important roles in bladder cancer tumorigenesis, progression, and drug resistance. YAP
Correspondence: Tian-Wen Xu Department of Medical Oncology, The Second Affiliated Hospital of Fujian Medical University No 950, Donghai Street, Fengze District, Quanzhou, Fujian 362000, China Tel +86 I 38085254 II Email xutianwen53@I63.com 
activation protects bladder cancer cells from chemotherapy and radiation-induced DNA damage. ${ }^{9}$ Moreover, YAP can interact with Nrf2 to maintain the chemoresistance in bladder cancer. ${ }^{10}$ YAP-induced cell growth and migration in bladder cancer cells depends on transcriptional cofactor Mask2, ${ }^{11}$ which can form a complex with YAP on target-gene promoters and is critical for YAP to activate transcription of target genes and tissue growth. ${ }^{12}$

YAP/TAZ pathway plays an important role in the maintenance of CSC properties in various human cancers. For example, YAP can occupy the promoters of mammary stem cell signature gene to induce cancer stem cells in breast cancer. ${ }^{13}$ In addition, YAP induced by SOX2 is essential for CSC characteristics in osteosarcoma and glioblastoma. ${ }^{14}$ Taken together, this evidence indicates that YAP/TAZ plays critical roles in CSC maintenance and cancer progression. However, CSC-specific regulatory mechanisms of YAP/TAZ and Hippo pathway remain largely unknown.

In this study, we showed that YAP is upregulated in $\mathrm{ALDH}^{\text {high }}$ populations of bladder cancer cell line. Silencing of YAP led to impaired self-renewal ability in bladder cancer cells. RNAseq results demonstrated that YAP can regulate the expression of ALDH1A1 in bladder cancer cells. In summary, we demonstrate that YAP is required for the stem cell property of bladder cancer stem cells via regulation of ALDH1A1.

\section{Materials and methods Cell culture and transfection}

Human bladder cancer cell lines 5637 and SCaBER were obtained from the Cell Bank of Chinese Academy of Sciences (Shanghai, China). The 5637 cells were maintained in DMEM (Invitrogen, Shanghai, China), supplemented with $10 \%$ fetal bovine serum (Invitrogen, Shanghai, China) in an incubator with $5 \% \mathrm{CO}_{2}$ at $37^{\circ} \mathrm{C}$. Subsequently, $20 \mathrm{~mL}$ of $10 \mathrm{mM}$ scrambled control or YAP siRNAs were purchased from Santa Cruz Biotechnology (Santa Cruz, CA, USA) and were transfected into 5637 using the Lipofectamine 2000 transfection reagent (Invitrogen, Shanghai, China) according to the manufacturer's protocol. For YAP overexpression, cells were cultured to $40 \%$ confluence and then transfected with lentivirus-based human YAP expression constructs (addgene pGAMA-YAP).

\section{FACS analysis and isolation}

The ALDH enzymatic activity of 5637 cells was determined by ALDEFLUOR kit (Stem Cell Technologies, Shanghai, China), according to the manufacturer's guidance. The data were analyzed using FlowJo software (Tree Star Inc., Ashland, OR, USA).

\section{Sphere culture and colony formation}

\section{assay}

For sphere, 5637 and SCaBER cells were cultured in a stem cell growth medium containing DMEM/F12 basal media (Invitrogen, Shanghai, China), N2 and B27 supplements (Invitrogen, Shanghai, China), $20 \mathrm{ng} / \mathrm{mL}$ human recombinant epidermal growth factor, and $20 \mathrm{ng} / \mathrm{mL}$ basic fibroblastic growth factor in 24-well ultra-low attachment plates. Sphere numbers were counted 10 days after culture. For colonyforming assay, 1,000 cells were seeded into six-well culture plates and cultured for 14 days to allow colony formation. Colonies were then stained with $0.1 \%$ crystal violet for quantification.

\section{Quantitative real-time PCR analysis ( $\mathrm{PPCR}$ ) and RNA sequencing}

Total RNA was extracted from cells using the Trizol reagent (Invitrogen), and cDNA was synthesized from total RNA using the SuperScript III (Invitrogen) according to the manufacturer's guidance. qPCR was performed using the Real-Time Quantitative PCR SYBR Green kit (Takara, Tokyo, Japan). Primer sequences used were $\beta$-actin forward 5'-AGGGGCCGGACTCGTCATACT-3'; $\beta$-actin reverse 5'-GGCGGCACCACCATGTACCCT-3'; YAP forward 5'-TAGCCC TGCGTAGCCAGTTA-3'; YAP reverse 5'-TCATGCTTAGTCCACTGTCTGT-3'; ALDH1A1 forward 5'-GCACGCCAGACTTACCTGTC-3'; ALDH1A1 reverse 5'-CCTCCTCAGTTGCAGGATTAAAG-3'. The relative expression of each gene was calculated using the $2^{\Delta \Delta} \mathrm{CT}$ method relative to the expression levels of $\beta$-actin. For RNAseq library preparation, mRNA was then isolated, fragmented, and reverse-transcribed into the cDNA libraries with an mRNAseq preparation kit (Illumina, San Diego, CA, USA). Library quality was determined using Agilent Bioanalyzer 2100 (Agilent). The Illumina Hiseq 2000 platform was used for single-end sequencing of cDNA libraries. Alignment of reads was performed using Tophat 2 with the hg19 build of the human genome. Transcript assembly and differential expression genes were identified by using Cufflinks software (Seattle, WA, USA). A heat map of differentially expressed genes involved in glutathione metabolism was generated using R.

\section{Western blotting}

Sorted cells were collected and nuclear protein was extracted using Nuclear Extraction Protocol (Thermo Fisher Scientific, Shanghai, China). Total amounts of total protein $(30 \mu \mathrm{g})$ were separated using SDS-PAGE and transferred to a polyvinylidene fluoride membrane. The membranes were blocked 
in $5 \%$ nonfat milk for 1 hour and incubated at $4{ }^{\circ} \mathrm{C}$ overnight with primary antibodies: anti-CD133 (1:1,000, sc-101199, Santa Cruz), anti-histone H3 (1:5,000, Abcam, Shanghai, China). Signals were visualized after incubation with horseradish peroxidase-conjugated secondary antibody.

\section{Statistical analysis}

Statistical analysis was performed using GraphPad Prism 5.0 software. Data are represented as mean \pm SD. Significant differences between groups were compared using two-tailed Student's $t$-test. $P<0.05$ was considered statistically significant. $* P<0.05, * * P<0.01, * * * P<0.001$.

\section{Results}

\section{Nucleus YAP is enriched in ALDH ${ }^{\text {high }}$ population in bladder cancer cells}

YAP has been previously reported to play essential roles in cancer stem cells' regulation in various cancers. ${ }^{13,14}$ In 5637 bladder cancer cell line, the $\mathrm{ALDH}^{\text {high }}$ cells display increased colony formation, migration, invasion, and ability to differentiate. ${ }^{7}$ To investigate the possible role of YAP on bladder cancer stem cell regulation, we first isolated 5637 $\mathrm{ALDH}^{\text {high }}$ subpopulation as assessed by the Aldefuor assay (Figure 1A). Indeed, the expression of YAP was nuclearenriched in $5637 \mathrm{ALDH}^{\text {high }}$ subpopulation (Figure 1B).

\section{YAP knockdown inhibited the colony- forming ability and self-renewal properties of bladder cancer stem cells}

To explore the effect of YAP on the self-renewal ability in the bladder cancer stem cells, we transfected the 5637 cells with YAP siRNA (knockdown group) or scramble control (control group). First, we checked cells' proliferation; however, we did not detect any difference (data not shown). Colony formation assay was then performed to determine the self-renewal capacity of 5637 cells. Our results showed a significant decrease in number of colonies in YAP-knockdown group compared to the control group (Figure 2A and B).
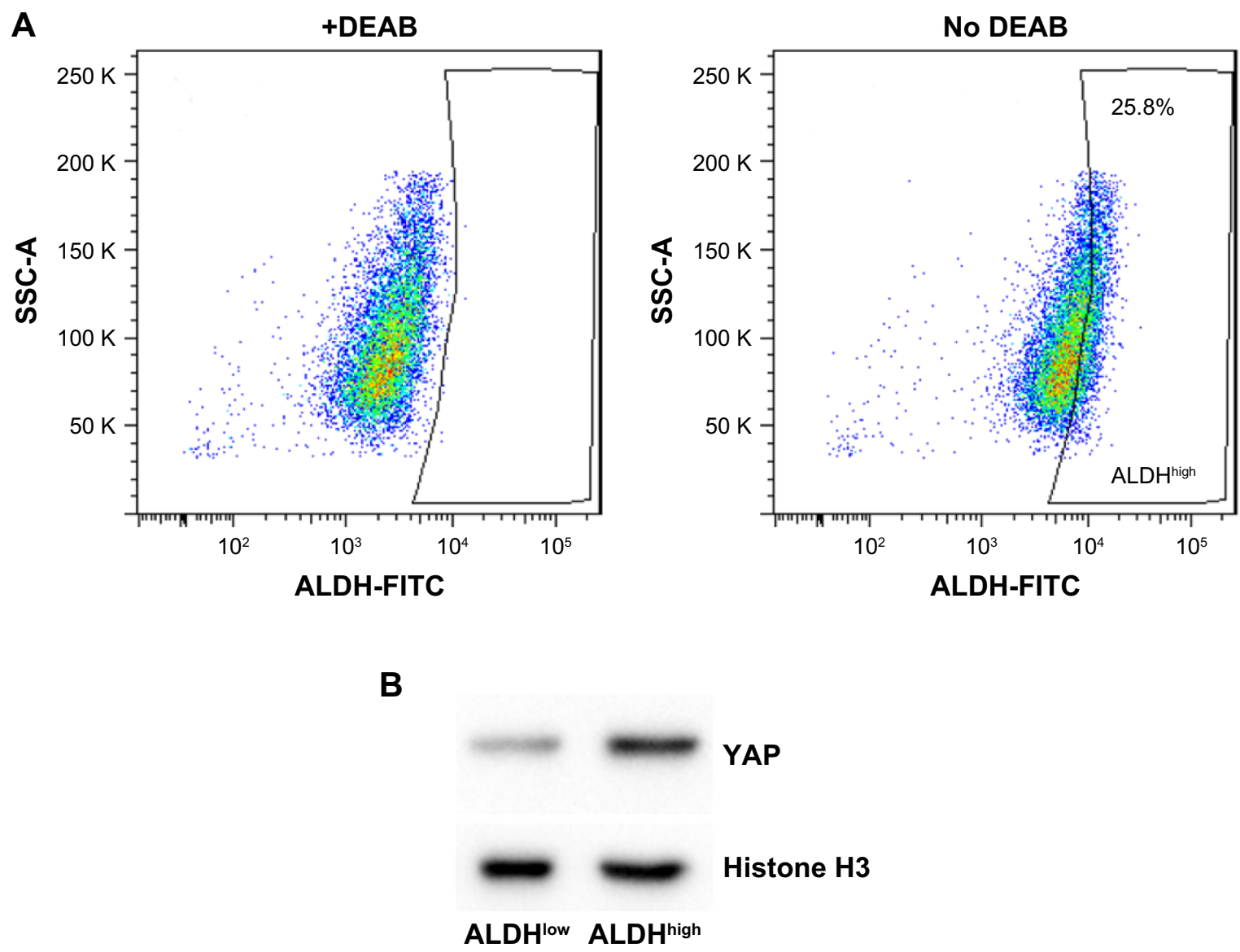

Figure I YAP expression is elevated in ALDH ${ }^{\text {high }}$ population.

Notes: (A) ALDH enzyme activity in 5637 cell line was analyzed by flow cytometry. Cells treated with the specific ALDH inhibitor DEAB were used as a negative control. The gated cells represent the ALDH high cells. (B) Western blot analysis of nucleus YAP protein levels in the ALDH ${ }^{\text {low }}$ and ALDH ${ }^{\text {high }}$ subpopulations from 5637 cells. Histone $\mathrm{H} 3$ was used as a loading control.

Abbreviations: $\mathrm{ALDH}^{\text {high, }}$, aldehyde dehydrogenase high; $\mathrm{ALDH}^{\text {low }}$, aldehyde dehydrogenase low; YAP, Yes-associated protein. 
A
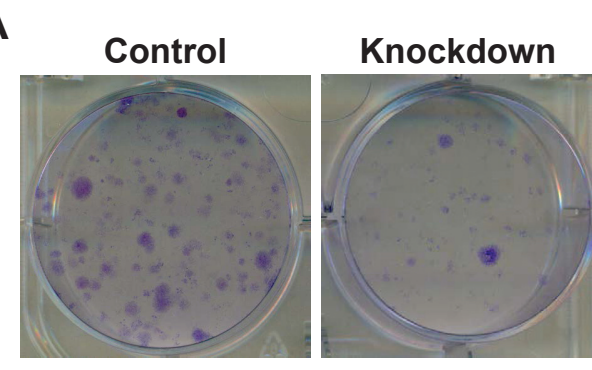

B

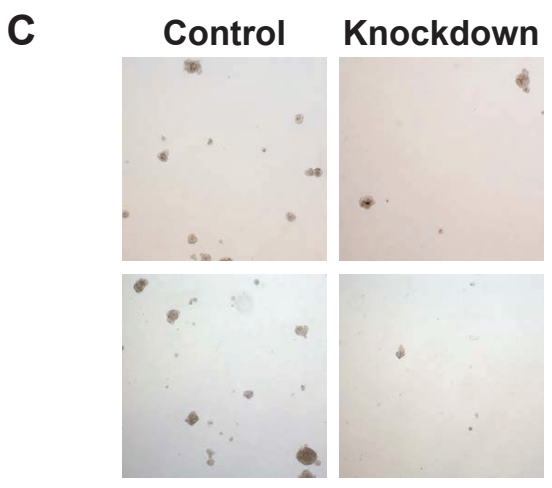

Figure 2 YAP is required for self-renewal capacity of bladder cancer cells.

Notes: (A, B) Colony-forming assays demonstrated that the clone formation ability of 5637 cells was significantly decreased in YAP knockdown group compared to the control group. (C) The representative photos of sphere formation of control and YAP knockdown bladder cancer 5637 and SCaBER cells. (D) The number of spheres was counted between control and YAP knockdown groups. $* * * P<0.001$. Data represent mean $\pm S D$ of triplicate experiments.

Abbreviations: CFU, colony-forming unit; YAP, Yes-associated protein.

ALDH ${ }^{\text {high }} 5637$ and SCaBER cells were further isolated by FACS sorting and cultured in stem cell medium for sphere assay. YAP siRNA (knockdown group) or scramble control siRNA were added to the medium to determine the function of YAP on sphere formation of bladder cancer stem cells. As shown in Figure 2C and D, our results clearly demonstrated that YAP silencing caused decrease of sphere formation of $\mathrm{ALDH}^{\text {high }} 5637$ cells. Our data suggested that YAP functions in regulating the self-renewal capacity of the 5637 cells.

\section{YAP is required for ALDH activity of bladder cancer}

We next asked the question whether YAP depletion in 5637 cells could lead to inhibition of ALDH ${ }^{\text {high }}$ subpopulation. To address that, we performed ALDH staining of both YAP knockdown and control 5637 cells. As shown in the flow cytometry results (Figure 3), silencing of YAP significantly inhibited the ALDH ${ }^{\text {high }}$ population of 5637 cells $(23.1 \%$ vs 4.3\%). This further supports that YAP is required for maintenance of cancer stem cell property in bladder cancer.

\section{YAP promotes self-renewal properties of bladder cancer stem cells}

To further validate the role of YAP on stemness of 5637 cells, we performed YAP overexpression assay to test the ability of YAP on sphere formation of bladder cancer stem cells. As shown in Figure 4A, our results showed that YAP protein was upregulated in overexpression 5637 cells. In addition, our sphere assay showed that overexpression of YAP resulted in increase of sphere formation of 5637 cells (Figure 4B and C). Again, our data further proved that YAP functions in regulating the self-renewal capacity of the 5637 cells.

\section{YAP is required for ALDHIAI expression of bladder cancer}

To further understand the downstream molecular events responsible for YAP regulation of bladder cancer stem cells, we extracted RNA from control and YAP knockdown 5637 cells and performed RNAseq. Our data showed that 343 transcripts were downregulated, and 143 transcripts were upregulated (fold change $>2 ; P<0.01$ ) (Figure 5A and Table S1). Among the most downregulated genes, we found that both YAP and ALDH1A1 were both significantly inhibited in the knockdown group (Figure 5A and Table S1). To verify our RNAseq results, we performed qPCR assay and found that depletion of YAP in 5637 cells significantly $(P<0.001)$ reduced expression of ALDH1A1 (Figure 5B). Based on these results, it is concluded that YAP activity is required for ALDH1A1 expression. 

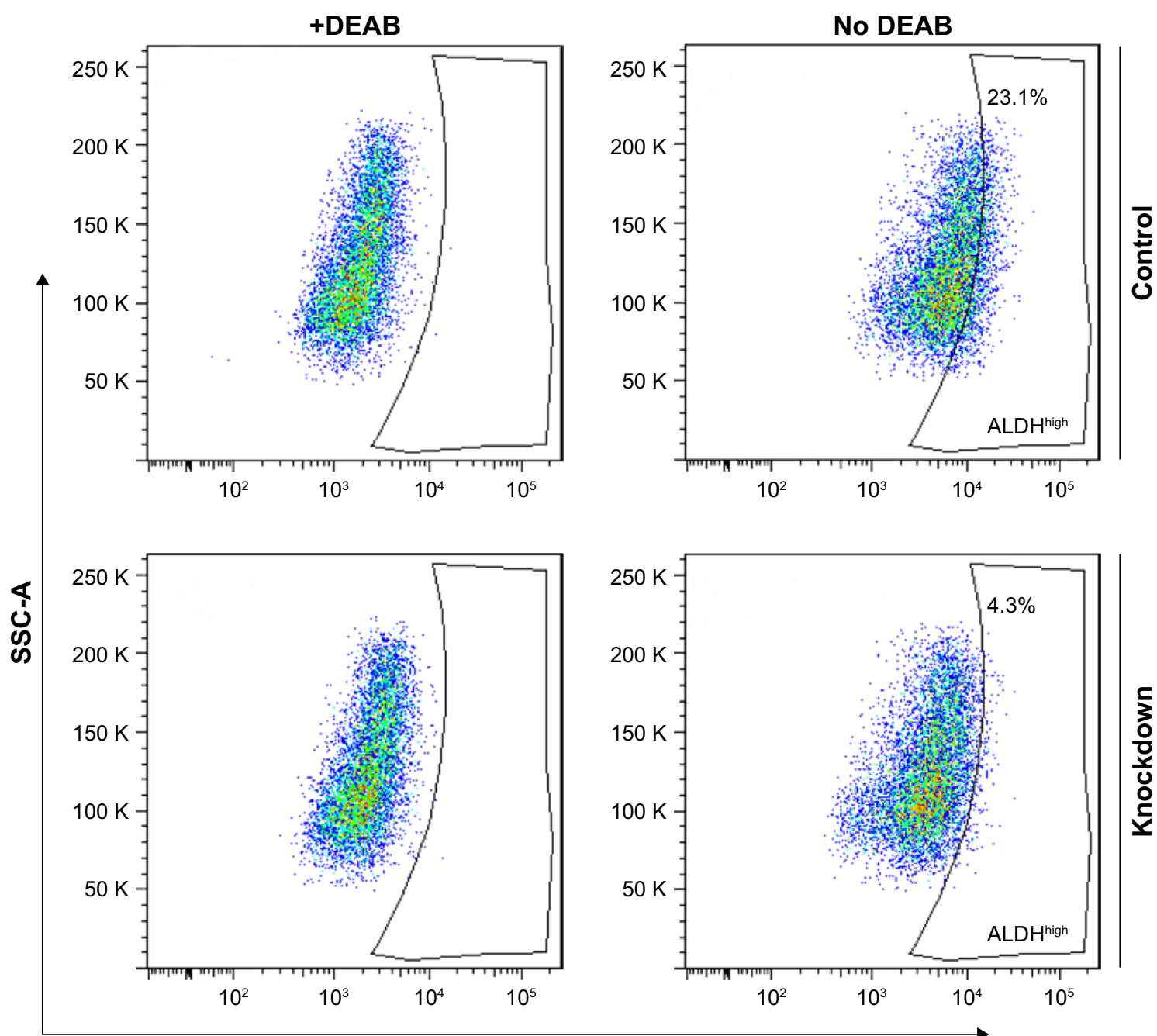

\section{ALDH-FITC}

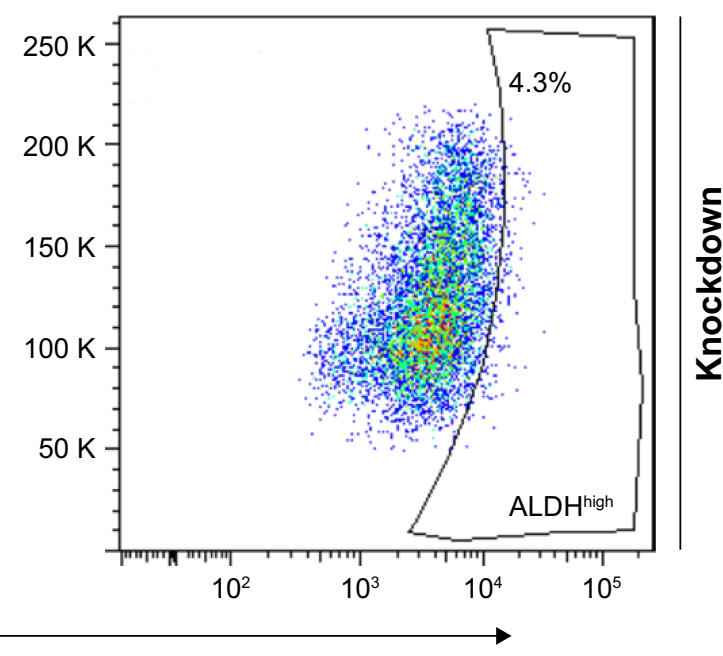

Figure 3 YAP is required for ALDH activity of bladder cancer cells.

Notes: The ALDH activity of the 5637 cells was analyzed after knockdown of YAP for 48 hours. The flow cytometry results demonstrated that the percentage of ALDH ${ }^{\text {high }}$ cells in the 5637 cells was dramatically inhibited upon depletion of YAP.

Abbreviations: ALDH, aldehyde dehydrogenase; FITC, fluorescein isothiocyanate; YAP, Yes-associated protein.

\section{Discussion}

Cancer stem cells play key roles in drug resistance, metastasis, and recurrence, which are responsible for the majority of cancer mortality. ${ }^{15}$ Hence, identifying cancer stem cells-related molecular mechanisms and characteristics is clinically important for developing better-targeted anticancer approach. The Hippo pathway regulates cell fate and stem cell differentiation during normal organogenesis and in the tumorigenesis. ${ }^{8}$ Our study provides strong evidence that YAP is required for ALDH1A1 expression and the properties of cancer stem cells in bladder cancer, thus suggesting that YAP might be a promising target in the prevention and treatment of bladder cancer.

Hyperactivation or gene amplification of YAP and TAZ is commonly found in various types of cancer, ${ }^{8}$ suggesting that YAP/TAZ is important for the development of neoplasia. In bladder cancer, previous study has shown that overexpression of YAP can promote cell growth and migration. ${ }^{11}$ In addition, YAP/Nrf2 crosstalk is critical for bladder cancer chemoresistance. ${ }^{10}$ In this study, we found that YAP is enriched in ALDH ${ }^{\text {high }}$ population of 5637 cells, suggesting that YAP/TAZ pathway might serve as an oncogenic driver that confers cancer stem cell traits in bladder cancer.

Interestingly, we found that YAP might regulate the properties of cancer stem cells of bladder cancer via regulation of ALDH1A1 gene expression. However, whether YAP can directly regulate ALDH1A1 expression needs further validation. Using in silico analysis, we found that several potential YAP/TEAD binding sequences locate within the promoter regions of ALDH1A1 genes. It will be worthwhile to validate whether YAP can directly bind to these sequences using ChIP assay. In addition to ALDH1A1 regulation, the 
A

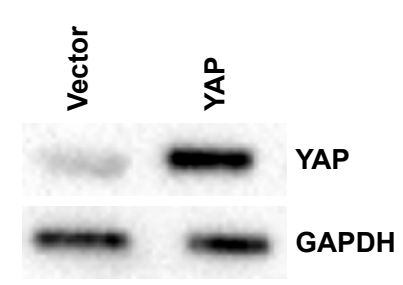

B

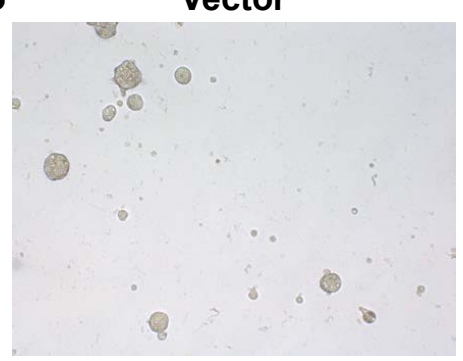

YAP

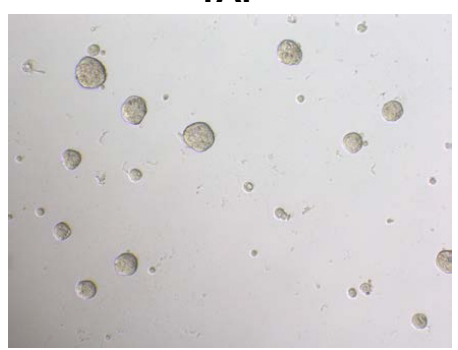

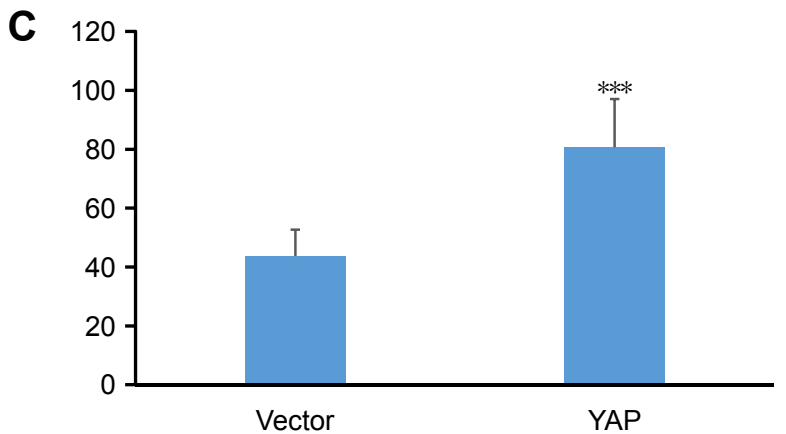

Figure 4 YAP promotes self-renewal properties of bladder cancer stem cells.

Notes: (A) Western blot analysis of YAP protein levels in the control and YAP overexpression 5637 cells. Glyceraldehyde 3-phosphate dehydrogenase was used as a loading control. (B) The representative photos of sphere formation of control and YAP overexpression 5637 cells. (C) The number of spheres was counted between control and YAP overexpression groups. ${ }^{* * *} P<0.001$. Data represent mean $\pm S D$ of triplicate experiments.

Abbreviation: YAP, Yes-associated protein.

Hippo pathway has also been shown to regulate epithelialmesenchymal transition and chemoresistance, suggesting alternate routes of YAP in regulating the properties of cancer stem cells. Indeed, our RNAseq data showed that many differentially expressed genes are involved in negative regulation of apoptotic process, suggesting that YAP might be important in antiapoptotic pathway. Despite its relevance in tumorigenesis, mutations in YAP are relatively
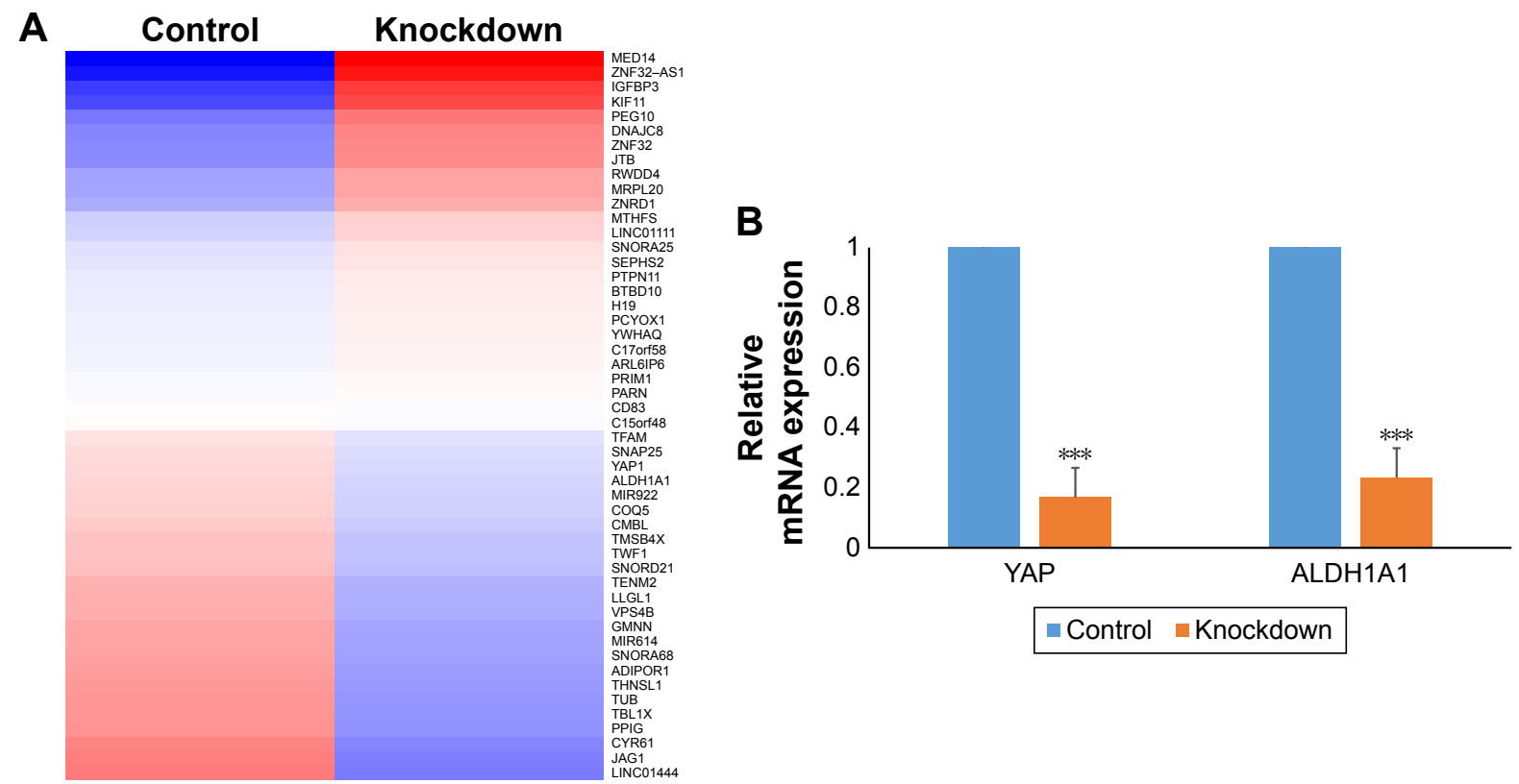

Figure 5 ALDHIAI expression depends on YAP in bladder cancer cells.

Notes: (A) Heatmap of 50 most differentially expressed genes between control and YAP knockdown 5637 cells. (B) Real-time PCR results confirmed that YAP and ALDHIAI mRNA expressions were inhibited in YAP knockdown 5637 cells. $* * * P<0.00$ I.

Abbreviation: YAP, Yes-associated protein. 
limited. Combination of conventional therapy and inhibition of YAP/TAZ may be promising approaches to target cancer stem cells.

In summary, we found that YAP is upregulated in bladder cancer stem cells and is essential for self-renewal ability of bladder cancer stem cells. We delineated a YAP/ALDH1A1 pathway in regulating bladder cancer stem cells and have showed that these interactions might have important clinical implications.

\section{Acknowledgments}

We thank our colleagues in Department of Medical Oncology, The Second Affiliated Hospital of Fujian Medical University for their critique during manuscript preparation.

\section{Disclosure}

The authors report no conflicts of interest in this work.

\section{References}

1. van Kessel KE, Zuiverloon TC, Alberts AR, Boormans JL, Zwarthoff EC. Targeted therapies in bladder cancer: an overview of in vivo research. Nat Rev Urol. 2015;12(12):681-694.

2. Rouanne M, Loriot Y, Lebret T, Soria JC. Novel therapeutic targets in advanced urothelial carcinoma. Crit Rev Oncol Hematol. 2016;98: 106-115.

3. Yang Z, Li C, Fan Z, et al. Single-cell sequencing reveals variants in ARID1A, GPRC5A and MLL2 driving self-renewal of human bladder cancer stem cells. Eur Urol. 2017;71(1):8-12.
4. Hatina J, Parmar HS, Kripnerova M, Hepburn A, Heer R. Urothelial carcinoma stem cells: current concepts, controversies, and methods. Methods Mol Biol. 2018;1655:121-136.

5. Fang D, Kitamura H. Cancer stem cells and epithelial-mesenchymal transition in urothelial carcinoma: possible pathways and potential therapeutic approaches. Int J Urol. 2018;25(1):7-17.

6. Chen $\mathrm{D}, \mathrm{Wu} \mathrm{M}, \mathrm{Li} \mathrm{Y}$, et al. Targeting $\mathrm{BMI}^{+}$cancer stem cells overcomes chemoresistance and inhibits metastases in squamous cell carcinoma. Cell Stem Cell. 2017;20(5):621-634.

7. Falso MJ, Buchholz BA, White RW. Stem-like cells in bladder cancer cell lines with differential sensitivity to cisplatin. Anticancer Res. 2012; 32(3):733-738.

8. Moroishi T, Hansen CG, Guan KL. The emerging roles of YAP and TAZ in cancer. Nat Rev Cancer. 2015;15(2):73-79.

9. Ciamporcero E, Shen H, Ramakrishnan S, et al. YAP activation protects urothelial cell carcinoma from treatment-induced DNA damage. Oncogene. 2016;35(12):1541-1553.

10. Ciamporcero E, Daga M, Pizzimenti S, et al. Crosstalk between Nrf2 and YAP contributes to maintaining the antioxidant potential and chemoresistance in bladder cancer. Free Radic Biol Med. 2018;115:447-457.

11. Dong L, Lin F, Wu W, Huang W, Cai Z. Transcriptional cofactor Mask2 is required for YAP-induced cell growth and migration in bladder cancer cell. J Cancer. 2016;7(14):2132-2138.

12. Sidor CM, Brain R, Thompson BJ. Mask proteins are cofactors of Yorkie/YAP in the Hippo pathway. Curr Biol. 2013;23(3):223-228.

13. Kim T, Yang SJ, Hwang D, et al. A basal-like breast cancer-specific role for SRF-IL6 in YAP-induced cancer stemness. Nat Commun. 2015; 6:10186.

14. Basu-Roy U, Bayin NS, Rattanakorn K, et al. Sox2 antagonizes the Hippo pathway to maintain stemness in cancer cells. Nat Commun. 2015; 6:6411.

15. Shibue T, Weinberg RA. EMT, CSCs, and drug resistance: the mechanistic link and clinical implications. Nat Rev Clin Oncol. 2017;14(10): 611-629.
OncoTargets and Therapy

\section{Publish your work in this journal}

OncoTargets and Therapy is an international, peer-reviewed, open access journal focusing on the pathological basis of all cancers, potential targets for therapy and treatment protocols employed to improve the management of cancer patients. The journal also focuses on the impact of management programs and new therapeutic agents and protocols on

\section{Dovepress}

patient perspectives such as quality of life, adherence and satisfaction. The manuscript management system is completely online and includes a very quick and fair peer-review system, which is all easy to use. Visit http://www.dovepress.com/testimonials.php to read real quotes from published authors. 